\title{
THE EQUATION OF STATE FOR A MODEL FERMI FLUID
}

\author{
J.C. OWEN \\ Department of Theoretical Physics, University of Manchester, Manchester M13 9PL, UK \\ and \\ R.F. BISHOP and J.M. IRVINE \\ Department of Theoretical Physics, University of Manchester, Manchester M13 9PL, UK, and \\ Daresbury Laboratory, Science Research Council, Daresbury, Warrington WA4 4AD, UK
}

Recelved 22 July 1976

Revised version recelved 20 October 1976

\begin{abstract}
A method of lowest order constrained variation advocated by the present authors and previously applied with excellent results to a model Boltzmann problem is generalized. New constraints are formulated for general Ferm 1 systems, and comparable success is demonstrated for the model Fermi problem.
\end{abstract}

In earlier publications $[1,2]$ we have elaborated a scheme for calculating the binding energy for manybody systems by a method of lowest order constrained variation (LOCV), which seeks to redress the objections open to the alternative lowest-order approach of Pandharipande [3,4].

We have demonstrated that our approach yields excellent agreement with the essentially exact results [5] for the Bethe "homework problem" over a wide density range. This problem comprises a hypothetical system of neutrons interacting via the repulsive part of the Reid soft-core ${ }^{1} \mathrm{~S}_{0}$ potential (acting in all partial waves),

$V(r)=6484.2 \mathrm{e}^{-7 x} / x \mathrm{MeV}$,

with $x=0.7 r$, and $r$ measured in fm. Additionally, Boltzmann rather than Fermi statistics are assumed. Since excellent agreement has now been reached, it seems appropriate to define a new homework problem where the particles obey Fermi statistics. To this end, and since there is still a demand for a reliable loworder approach for application to such real systems as nuclear matter or finite nuclei with realistic potentials, we generalize in this work our previous $[1,2]$ method, and impose new constraints suited to dense Fermi systems. The new method is then tested on the Fermi homework problem, and compared with other recent results $[6,10]$.

As before we adopt a trial wave function,
$\Psi=\prod_{i<j} f(i) \Phi$,

where $\Phi$ is the (uncorrelated) ground-state wavefunction of $N$ independent fermions of spin-1sospin degeneracy $\nu$ and mass $m$, in a volume $\Omega$ with number density $\rho=N / \Omega=\nu k_{\mathrm{F}}^{3} / 6 \pi^{2}$, and Fermi wavenumber $k_{\mathrm{F}}$. As appropriate, the two-body potential and the correlation function, both of which are considered momentum-independent, can be decomposed into relative partial-wave components $V_{l}(r)$ and $f_{l}(r)$.

Our previous $[1,2]$ LOCV prescription involved truncation of the Jastrow cluster series for the energy at the two-body level, but with constraints imposed on the pair correlation function $f(r)$ which are devised to justify this truncation. These constraints assume the form of a certain bound,

$0 \leqslant f_{l}(r) \leqslant h_{l}(r)$,

and an integral inequality of the form,

$4 \pi \rho \sum_{l} \int_{0}^{\infty} G_{l}\left[f_{l}(r), h_{l}(r)\right] \mathrm{d} r \leqslant I$,

with prescribed forms of the functions $h_{l}$, the functionals $G_{l}$, and the parameter $I$. In addition, the functionals $G_{l}$ satisfy the condition,

$G_{l}\left[h_{l}(r), h_{l}(r)\right] \equiv 0$.

The two-body energy in the cluster expansion can be expressed as, 
Table 1

Energy per particle versus density using different constraints.

\begin{tabular}{|c|c|c|c|c|c|c|c|c|}
\hline \multirow{2}{*}{$\begin{array}{l}\rho \\
\left(f m^{-3}\right)\end{array}$} & \multicolumn{4}{|c|}{$E / N(\nu=2)(\mathrm{MeV})$} & \multicolumn{4}{|c|}{$E / N(\nu=4)(\mathrm{MeV})$} \\
\hline & LOCVN1 & LOCVN2 & LOCVB1 & LOCVB2 & LOCVN1 & LOCVN2 & LOCVB1 & LOCVB2 \\
\hline 0.2 & 98.35 & 92.03 & 86.13 & 83.36 & 89.06 & 87.37 & 7860 & 76.45 \\
\hline 0.4 & 227.3 & 2229 & 183.7 & 213.1 & 213.1 & 210.3 & 181.4 & 177.8 \\
\hline 0.6 & 386.8 & 381.8 & 311.8 & 306.4 & 368.3 & 364.5 & 309.7 & 3051 \\
\hline 0.8 & 569.7 & 562.5 & 455.9 & 488.6 & 548.6 & 543.7 & 458.0 & 453.8 \\
\hline 1.0 & 772.0 & 766.0 & 616.4 & 608.8 & 747.7 & 741.8 & 626.5 & 620.8 \\
\hline 1.2 & 990.7 & 982.2 & 791.6 & 783.9 & 962.6 & 958.3 & 808.4 & 801.8 \\
\hline 1.4 & 1223 & 1217 & 979.5 & 970.2 & 1191 & 1187 & 1003 & 994.5 \\
\hline 1.6 & 1467 & 1460 & 1179 & 1170 & 1437 & 1427 & 1209 & 1204 \\
\hline
\end{tabular}

$E_{2} / N=2 \pi \rho \sum_{l} \int_{0}^{\infty}\left[\hbar^{2} m^{-1} f_{l}^{\prime 2}(r)+V_{l}(r) f_{l}^{2}(r)\right] a_{l}^{2}(r) \mathrm{d} r$

where the functions $\phi_{l}^{2}\left(k_{\mathrm{F}} r\right) \equiv a_{l}^{2}(r) / r^{2}$ are proportional to the squares of the partial-wave components of the antisymmetrized uncorrelated fermion pair wavefunctions, averaged over the Fermi sea. The total energy per particle also contains a one-body kinetic energy of $\left(\frac{3}{10}\right) \hbar^{2} k_{\mathrm{F}}^{2} / m$, which is included in the numerical results. In the present case the potential $V_{l}(r)$ $1 \mathrm{~s}$ independent of $l$, and we shall present results for the two cases where the correlation function $f(r)$ is either state-independent (1.e. independent of $l$ ), or at most depends only on the channel parity, 1.e.

$f(r)=f_{+}(r) P_{+}+f_{-}(r) P_{-}$,

where $P_{ \pm}$are projectors on to the even and odd partial waves respectively. In these cases, the summation over $l$ in eq. (6) can be simplified, using the results,

$$
\begin{aligned}
& \frac{1}{2} \sum_{l}\left[1 \pm(-1)^{l}\right] \phi_{l}^{2}(x) \equiv a_{ \pm}^{2}(r) / r^{2} \\
& \quad=(\nu \mp 1) /(2 v)\left[1 \pm 9 x^{-2} j_{1}^{2}(x)\right], a^{2}(r) \equiv a_{+}^{2}(r)+a_{-}^{2}(r),
\end{aligned}
$$

where $x \equiv k_{F} r$. We have shown [2] that eq. (6) can be minimized subject to the constraints (3)-(5) as follows. The solutions to each of the Euler-Lagrange equations,

$g_{t}^{\prime \prime}-\left\{\frac{a_{t}^{\prime \prime}}{a_{t}}+\frac{m}{\hbar^{2}} V_{t}\right\} g_{t}+\frac{m \lambda}{2 \hbar^{2} a_{t}} \frac{\delta G_{t}}{\delta f_{t}}=0$,

where $g_{t}(r) \equiv a_{t}(r) f_{t}(r)$, are first obtaned in the range $0 \leqslant r \leqslant d_{t}$, where the correlation distances $d_{t}$ are given by $f_{t}^{\prime}\left(d_{t}\right)=h_{t}^{\prime}\left(d_{t}\right)$; and for $r \geqslant d_{t}$ we set $f_{t}(r)=$ $h_{t}(r)$. The index $t$ ranges over the number of distinct correlation functions used. The constant $\lambda$ in eq. (8), which is the Lagrange multiplier for constraint (4), is either set to zero if this constraint is thus satisfied as an inequality, or is varied iteratively until it is satisfied as an equality.

Previously $[1,2,7]$ we have used the above constraints in the form,

$h_{t}(r)=1, I=1, G_{t}\left[f_{t}, h_{t}\right]=\left[h_{t}^{2}(r)-f_{t}^{2}(r)\right] a_{t}^{2}(r)$.

The first of these constraints has been justified for Bose systems on the basis that it is unreasonable to allow the long-range behaviour of the potential to have a large effect on the two-body correlation function. Similarly the other two constraints, which imply that the correlations do not expel more than one particle from within their mutual range of strong influence (i.e. from within the correlation distances), seem to be a natural choice for consistency within a two-body approximation.

On the other hand, for Fermi systems, the Pauli exclusion principle induces short-range correlations in the system even in the absence of interactions. This is reflected in the suppression near the origin of the factor $a^{2}(r)$ of eq. (7) (assuming state-independence) in comparison with its Bose counterpart $(1-1 / N) r^{2}$; and hence constraint (4) becomes a weaker constraint for fermions than for bosons. Accordingly we shall reformulate the constraints (9) for fermions, but in such a manner that for bosons they remain unchanged.

We first note that for bosons, the minimum value of $E_{2} / N$ from eq. (6) is changed only by $O(1 / N)$ if the first of eqs. (9) is changed to read $h_{t}(r)=$ $(1-1 / N)^{-1 / 2}$. This purely formal procedure shows that constraint (4) now reads, 


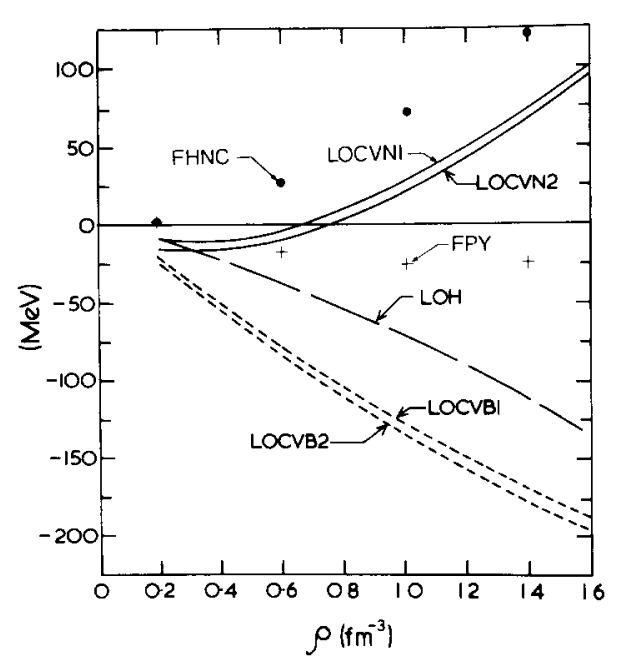

Fig. 1 Comparison of the results of several approximations for the $\nu=2$ Fermı homework problem, displayed as $E / N)_{\mathrm{X}}$ $-E / N)_{\mathrm{KT}}$, where the label $\mathrm{x}$ shown in the figure indicates the approximation used. The KT and $\mathrm{LOH}$ results are taken from ref. [6], the FHNC and FPY results are taken from ref. $[10]$, and the remainder from the present calculations

$\rho \int\left\{1-f^{2}(r) \phi^{2}\right\} \mathrm{d} r \leqslant I$.

Taking the equality sign and $I=1$ as before, eq. (10) merely expresses the two-body approximation to the normalization condition, which must be satisfied by any exact radial distribution function [8]. Since it is known that imposition of the normalization condition has the effect of stimulating rapid convergence of the energy cluster series [9], this may indicate why constraints (9) give such demonstrably good results for bosons.

Employing a similar procedure for fermions leads by analogy to the choice,

$h_{t}(r)=r / a(r), \quad I=1$,

$G_{t}\left[f_{t}, h_{t}\right]=\left[h_{t}^{2}(r)-f_{t}^{2}(r)\right] a_{t}^{2}(r)$,

where $a(r)$ is given by eq. (7), and use of eq. (7) easily shows that this choice satisfies the Fermi analogue of the normalization condition (10). A simple rearrangement and a trivial integration also shows that one of the convergence parameters [2], $k$, associated with the cluster series,

$k=4 \pi \rho \sum_{l} \int_{0}^{\infty}\left[1-f_{l}^{2}(r)\right] a_{l}^{2}(r) \mathrm{d} r$,

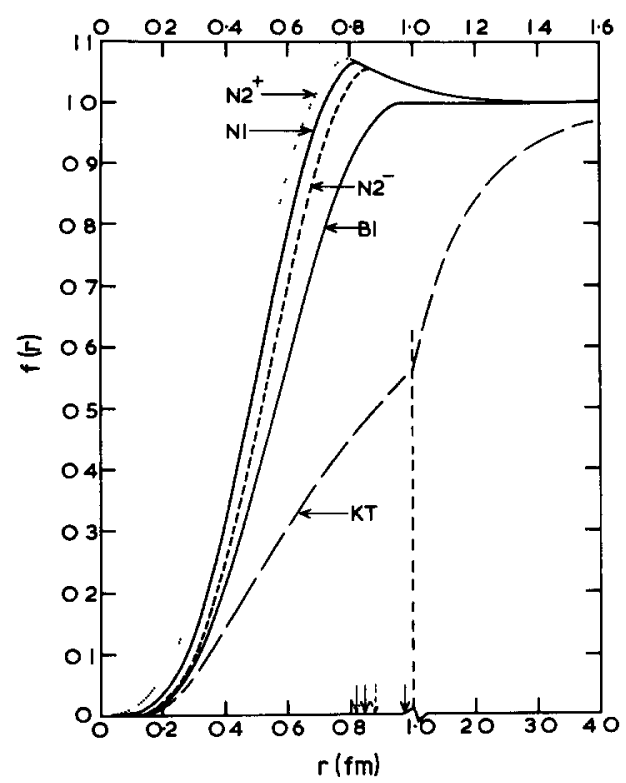

Fig. 2. The correlation functions $f(r)$ of several approximations for the $\nu=2$ Fermi homework problem at a density $\rho=1.0$ $\mathrm{fm}^{-3}$. The arrows indicate the correlation distances $d_{t}$ for the present calculations. Notice the change of scale at $r=1.0 \mathrm{fm}$, applicable only to the KT result taken from ref. [6].

is identically zero when the integral constraint of eqs.

(4) and (11) operates.

We present in table 1 results obtained by the method outlined above for fermions of either degeneracy $\nu=2$ or 4 , interactıng via the homework potential of eq. (1), and employing constraints (3)-(5) with both the forms expressed in eq. (9) as well as in eq. (11). We refer to these constraints as boson constraints and normalization constraints respectively, and the corresponding methods are labelled LOCVB $n$ and LOCVN $n$, where the additional integer $n$ indicates the number of distinct correlation functions employed, i.e. the range of the label $t$ in eq. (8).

Krotscheck and Takahashi (KT) [6] perform an unconstrained variational calculation for the same $\nu=2$ Fermı problem, with a single parametrized correlation function of assumed analytic form, summing an infinite partial series of cluster contributions which reduce to those included in the hypernetted chain approximation in the limit of Bose statistics. We compare our results in fig. 1 with the most ambitious calculations of KT which attempt to go beyond the lowest order hypernetted chain ( $\mathrm{LOH})$ approximation by inclusion of the leading basic diagrams, but we also show their $\mathrm{LOH}$ results for comparison. 
In a very recent communication, Fantoni and Rosati (FR) [10] also examine the same model Ferm1 problem using their Fermi hypernetted chain (FHNC) and Fermi Percus-Yevick (FPY) formalisms, wherein the dynamic and statistical correlations are both treated to the same level. In particular the FHNC method takes into account all cluster diagrams contributing to the radial distribution function except those containing elementary subdiagrams. In their calculations, FR also use a state-independent Jastrow correlation function of the same single-parameter analytic form used by both KT [6], and Chakravarty et al. (CMW) [11] for the corresponding model Bose problem, but FR have not optimised this choice by minimising the energy at each density with respect to variations in this parameter. They give FHNC results using the "optimum" correlation functions obtained by both KT [6] for the model Fermi liquid, and by CMW [11] for the corresponding model Bose liquid. The differences in energy so obtained, whilst small $(\approx 1-2 \%)$, indicate that optimızation might well decrease their results still further by a few percent. These results are also displayed in fig. 1. (Note that we have used their FHNC values obtained with the CMW correlation functions, since these lie lower in energy.)

Of the methods displayed in fig. 1, the FHNC results are certainly the most ambitious in that they sum the largest class of cluster diagrams, and until each has been checked for convergence by including more terms (e.g. by extending FHNC to FHNC/4, etc.), they should probably presently be regarded as the most reliable. On the other hand, the difference between the KT and FHNC results can probably be regarded as an indication of the remaining uncertainty.

The following points should be noted: (i) As expected our LOCVN results are in markedly better agreement with both the KT and FHNC results than our LOCVB results, or indeed than the LOH results. Even at nearly ten tımes nuclear matter density, the LOCVN results agree to within $7 \%$ with the KT results and to within $4 \%$ with the FHNC results, whereas the LOCVB results are out by $15-20 \%$ even with the KT results. In addition the LOCVN results lie above the KT results for most densities, by contrast with the LOCVB and LOH results which always lie below; and although all other results lie below the FHNC results, it should again be remembered that these have not yet been optimised. (ii) By contrast with the LOCVB results, the LOCVN results now bear qualitatively the same relationship to the $\mathrm{KT}$ results as did our previous results with Boltzmann statistics to the comparable "exact" Bose results of CMW [11]; and although the LOCVN results lie lower than the FHNC results, their overall agreement is excellent. (iii) Allowing state-dependence into the correlation function typically gives a decrease in energy of about 5-8 MeV per particle, and although we clearly do not trust our methods to anything approaching this accuracy, we believe this difference is an accurate indicator of the state-dependence to be expected in the more ambitious calculations. (1v) Finally we compare in fig. 2 the correlation functions obtained by our method with that obtained in the unconstrained KT calculation. The long-range behaviour of the LOCVN correlation functions, which is dictated by the first constraints (11) is now characterised by an "overshoot", although this is seen to be small and of quite short range in comparison with the $\mathrm{KT}$ result.

In conclusion, these results indicate that LOCV calculations with properly chosen constrants can probably be made as successful for Fermi as for Boltzmann systems, at least for repulsive short-range potentials of the type considered here. Comparable agreement with "exact" results is also found for other more "realistic" model potentials including attraction. Further details of the present method and applications to such other models will appear elsewhere [12].

\section{References}

[1] J.C. Owen et al., Phys. Lett. 59B (1975) 1.

[2] J.C Owen, R.F. Bishop and J.M. Irvine, Ann. Phys. (NY), to be published.

[3] V.R Pandharipande, Nucl. Phys A174 (1971) 641.

[4] V R. Pandharipande, Nucl. Phys. A181 (1972) 33.

[5] L. Shen and C.-W. Woo, Phys. Rev. D10 (1974) 371.

[6] E. Krotscheck, K. Takahashi, Phys. Lett. 61B (1976) 393.

[7] J.C. Owen et al., Phys. Lett. 61B (1976) 147

[8] M.E. Grypeos and E. Mavrommatis, Lett. Nuovo Cim. 5 (1972) 369.

[9] G.P. Mueller, J.W. Clark, Nucl. Phys. A155 (1970) 561; M.L. Ristig et al., C3 (1971) 1504.

[10] S Fantonı, S. Rosati, Lett. Nuovo Cim. 16 (1976) 531.

[11] S. Chakravarty et al., Nucl. Phys. A220 (1974) 233.

[12] J.C. Owen, R.F. Bishop and J.M. Irvine, Nucl. Phys., to be published. 